\title{
Modal Analysis of Vertical Turbine Pump
}

\author{
A.Y.Nikumbe ${ }^{1}$, V.G.Tamboli ${ }^{2}$, H.S.Wagh ${ }^{3}$ \\ Student, Department of mechanical engineering, Sinhgad Institute of Technology,Lonavala, Pune, India ${ }^{1,2,3}$
}

\begin{abstract}
This paper discuses modal analysis of vertical turbine pump. Vertical Turbine Pumps are the centrifugal pumps which are mounted vertically. Generally, turbine pumps have constant head and fluid flows uniformly at high pressure. Natural frequencies of a vertical turbine pump are calculated by performing a modal analysis using the Finite Element Method (FEM). Common name for this type of analysis is Finite Element Analysis (FEA). Accuracy of these numeric methods depends on numbers of elements. The primary reason for conducting an FEA is to obtain an acceptable separation margin between the unit operating speed and the individual structural natural frequencies. Total six modes of vibration are found for this analysis. Experimental analysis is defined as the study of dynamic characteristics of a mechanical structure. Experimental analysis is done by using Fast Fourier Transform (FFT) analyzer. During this analysis, exciter mechanism is done by using an instrumental hammer, as this mechanism requires a minimum amount of hardware and provides shorter measurement times. At last, by comparing natural frequency with operational frequency of vertical turbine pump ensures the safe working of the pump.
\end{abstract}

Keywords: Vertical Turbine Pump (VTP), Modal Analysis, FEA, EMA.

\section{INTRODUCTION}

A pump is a device or an apparatus used for conveying a fluid from one point to other, usually through pipe. Pump can be also defined as a mechanical device which translates the mechanical energy imparted to it from external source (electric motor, diesel engine, or even manual energy) into hydraulic energy in the field handled by it.

A specialized centrifugal pump designed to move water from a well or reservoir that is deep underground are called vertical turbine pump. They are also known as deep well turbine pump or a line shaft turbine pump. The two main types of turbine pumps are vertical turbine pumps and submersible turbine pumps. Submersible pumps have the electric motor located underwater at the bottom of the pump, vertical turbine pumps have the motor located above ground, connected via a long vertical shaft to impellers at the bottom of the pump. Pumps are divided into 3 sections .viz. bowl assembly, head assembly and column assembly. These pumps operate on same principle like centrifugal pumps operate with a rotating impeller around the central shaft. The major advantage of turbine pump is its low revolutions per minute or rpm with high and constant head. The pressure head developed depends on diameter of impeller and the speed at which it is rotated.

Natural frequency means the number of cycles per minutes that the rotor or structure will vibrate as if it is "rapped" like a tuning fork. Structural natural frequencies of vibration and their mode shapes of a vertical turbine pump is predicted by performing a modal analysis using the Finite Element Method (FEM) by using the analysis software ANSYS(2011). The common name to refer to this kind of analysis is Finite Elements Analysis (FEA). The finite element method is a numerical method for solving problems of engineering and mathematics. In this method, a domain is divided into $\mathrm{n}$ numbers of sub domains, the elements, and equations of $\mathrm{n}$ grade are used to approximate the solutions of each sub domain. The summation of responses of all the elements makes up the total response of the problem. The accuracy of this numeric method depends on the number of sub domain into which the domain has been divided and the boundary conditions.

In this analysis, motor having near about 736 $\mathrm{rpm}$ is used. This selection is done on the basis of the required starting torque, environmental condition, efficiency, operating speed of the pump and size of drive.

Therefore, to find out the operating speed, convert this rpm speed into Hertz $(\mathrm{Hz})$ as given below,

$$
736 / 60=12.3 \mathrm{~Hz}
$$

Hence, the operating speed of the pump is $12.3 \mathrm{~Hz}$

Experimental modal testing is the form of vibration testing of an object in which the natural frequencies, modal masses, modal damping ratios and mode shapes of an object under test are determined. This testing is the study of dynamic characteristics of a mechanical structure. The testing is done by using Fast Fourier Transform (FFT) analyzer. A fast Fourier transform (FFT) consists of many types of excitation sources can be implemented to measure a structure's frequency response function. The excitation function is the mathematical signal used for the input. The excitation system is the physical mechanism used to prove the signal. Excitation can be done by many methods like impact hammer, shaker method etc.

Finally, comparison of both the experimental and modal analysis is done. 


\section{FINITE ELEMENT ANALYSIS (FEA)}

Finite Element Analysis(FEA) is a numerical technique for finding approximate solution to boundary value problems for partial differential equations.[2] It uses subdivision of a whole problem domain into simpler parts, called finite elements and variation methods like Galerkin method, Rung-Kutta method etc. Solution of modal analysis of vertical turbine pump by using FEA involves following steps.

A. Geometry:

All the parts of the vertical turbine pump are drawn by using Creo-Parametric (modelling software). in this, the parts are saved in the .prt format and the assembly is saved as .asm format. So if we want to import geometry in ANSYS Workbench firstly it should be converted into .igs format.

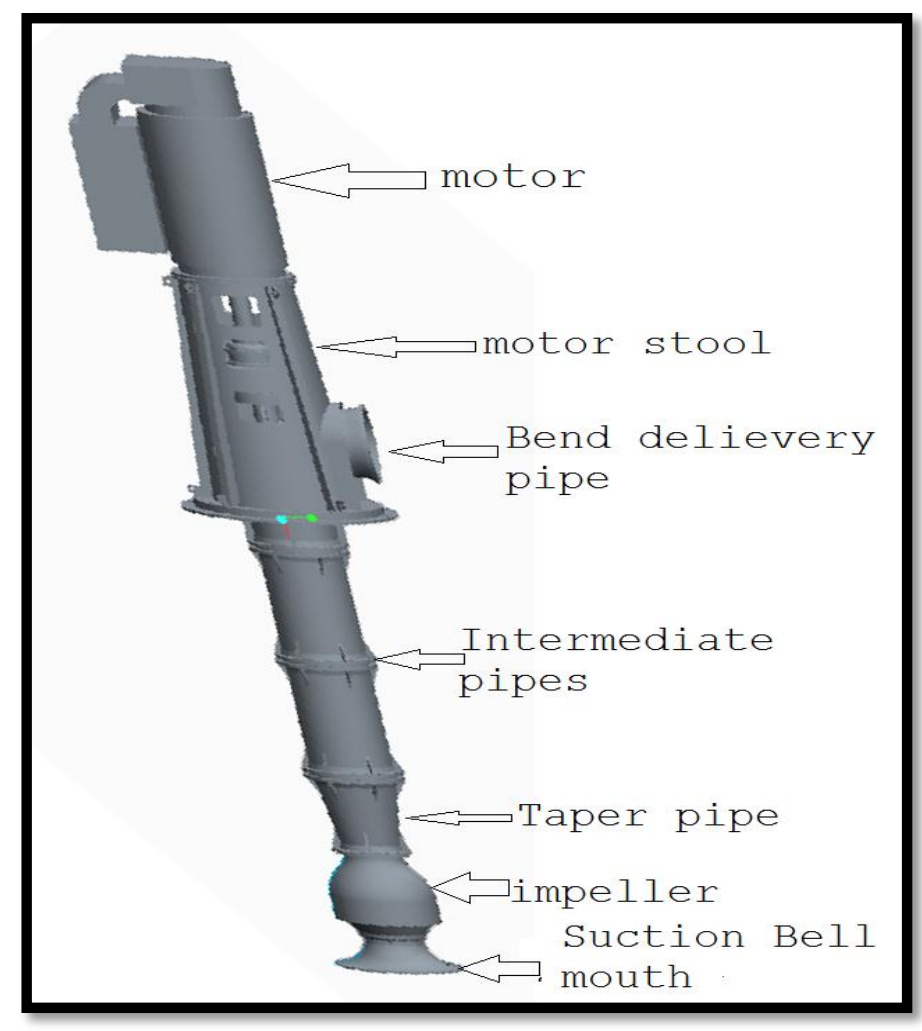

Fig1. Assembly of VTP

B. B. Meshing:

After the importation of the geometry material properties are assigned. Material properties like density (in $\mathrm{kg} / \mathrm{m}^{3}$ ), Poisson's ratio, young's modulus (in Pascal) are assigned to different parts of the vertical turbine pump.

table1. material data

\begin{tabular}{|l|l|c|c|c|}
\hline Material Name & Component of VTP & \multicolumn{1}{|c|}{$\begin{array}{c}\text { Density } \\
\left(\mathbf{k g} / \mathbf{m}^{\mathbf{3}}\right)\end{array}$} & $\begin{array}{c}\text { Young's } \\
\text { Modulus(Pa) }\end{array}$ \\
\hline $\begin{array}{l}\text { ASTM A439 TYPE } \\
\text { D2 }\end{array}$ & $\begin{array}{l}\text { Casing, Discharge } \\
\text { Bend, Pipes,etc }\end{array}$ & 7800 & 0.26 & $1.31 \mathrm{e}+011$ \\
\hline $\begin{array}{l}\text { ASTM A276 TYPE } \\
\text { XM-19A }\end{array}$ & Int. Brg. Sleeve & 7888.6 & 0.298 & $1.8 \mathrm{e} 011$ \\
\hline SS 316L & Strainer & 8000 & 0.28 & $2.0 \mathrm{e} 011$ \\
\hline $\begin{array}{l}\text { ASTM A276 TYPE } \\
\text { SS 316L }\end{array}$ & $\begin{array}{l}\text { Coupling, Shaft, } \\
\text { Key,etc }\end{array}$ & 7400 & 0.26 & $2.0 \mathrm{e} 011$ \\
\hline $\begin{array}{l}\text { IS:2062 Gr } \\
\text { FG260 }\end{array}$ & Brg. Housing & 7200 & 0.26 & $1.28 \mathrm{e} 011$ \\
\hline $\begin{array}{l}\text { ASTM A743 Gr } \\
\text { CA-15 }\end{array}$ & Brg. Cover & 7750 & 0.24 & $1.99 \mathrm{e} 011$ \\
\hline BRONZE & Motor & 1200 & 0.33 & $1.10 \mathrm{e} 011$ \\
\hline ASTM A216 WCB & $\begin{array}{l}\text { Flexible Spacer Type } \\
\text { Coupling }\end{array}$ & 7820 & 0.29 & $1.92 \mathrm{e} 011$ \\
\hline MS IS:2062 Gr B & $\begin{array}{l}\text { Sole Plate, Motor } \\
\text { Stool }\end{array}$ & 7850 & 0.30 & $2.1 \mathrm{e} 011$ \\
\hline
\end{tabular}


After assigning material properties, geometry is divided D. FEA results:

into numbers of finite elements. The process of subdivision of domain into finite elements called as The following bar chart Frequency vs. Modes indicates the Discretization process also known as Meshing. Model is frequency at each calculated mode.

meshed with hexagonal elements. Meshing is used to get accurate results.

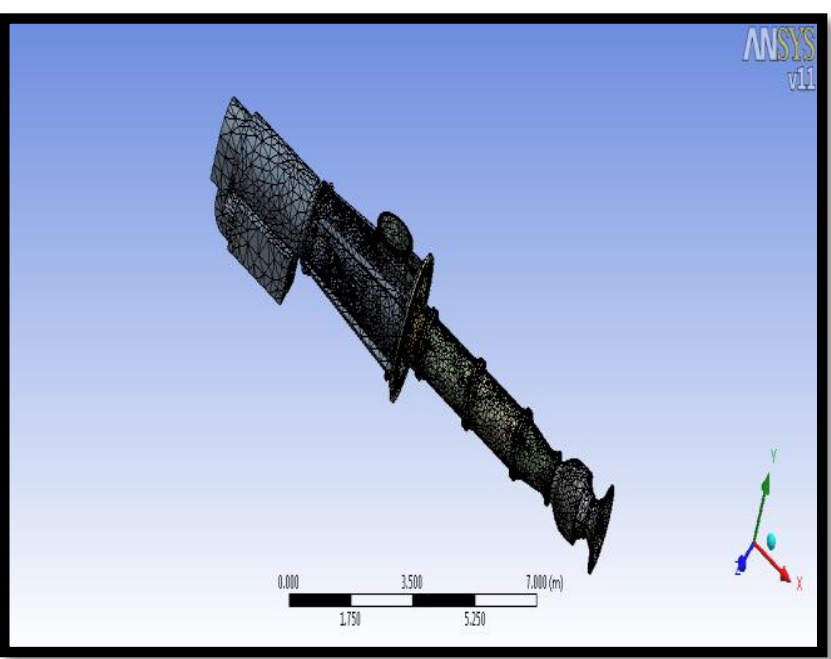

Fig 2. Mesh Model of VTP

\section{Simulation:}

In the simulation process, initial conditions are given to pump. In this, fixing of the sole plate is given as the initial condition as it is placed on the surface of the ground i.e. Base. After that geometry is going under process to get solution and results.

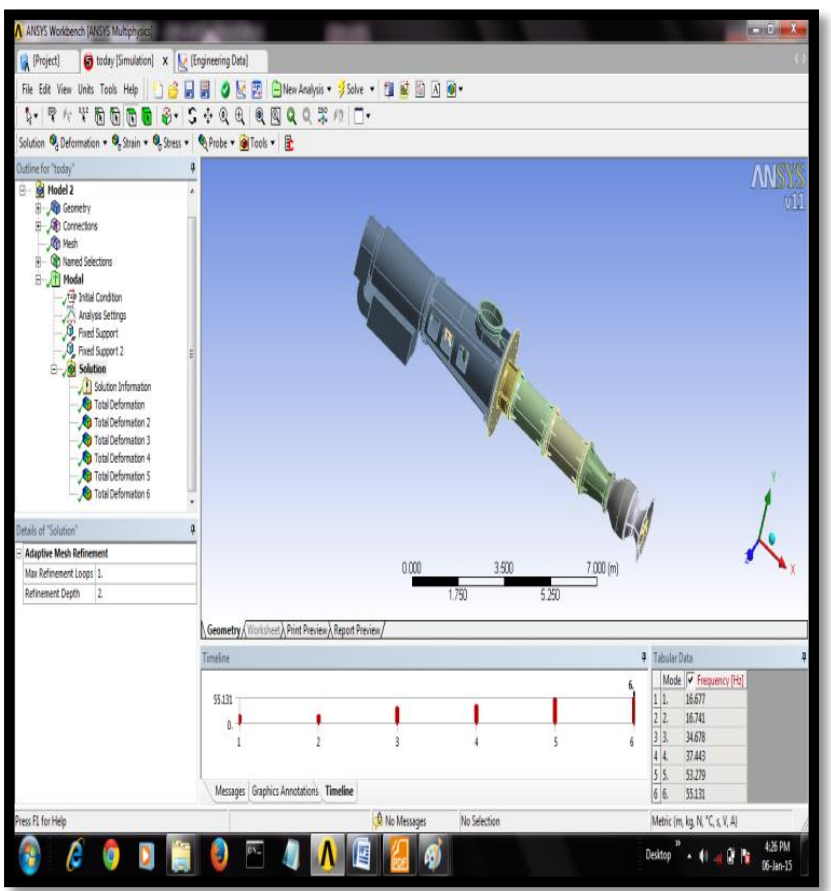

Fig.3 Simulation of VTP
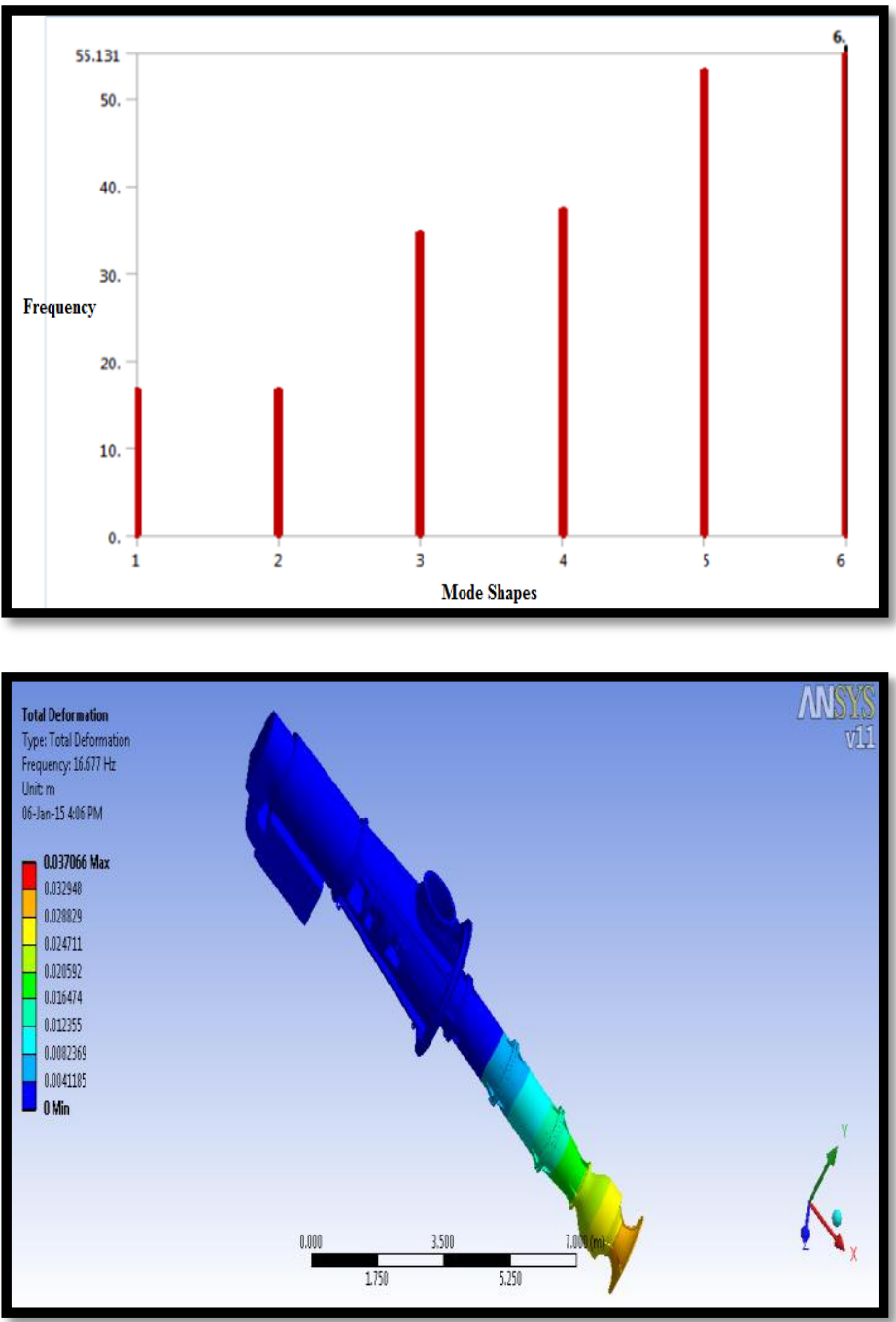

Fig 4(a) First Mode shapes

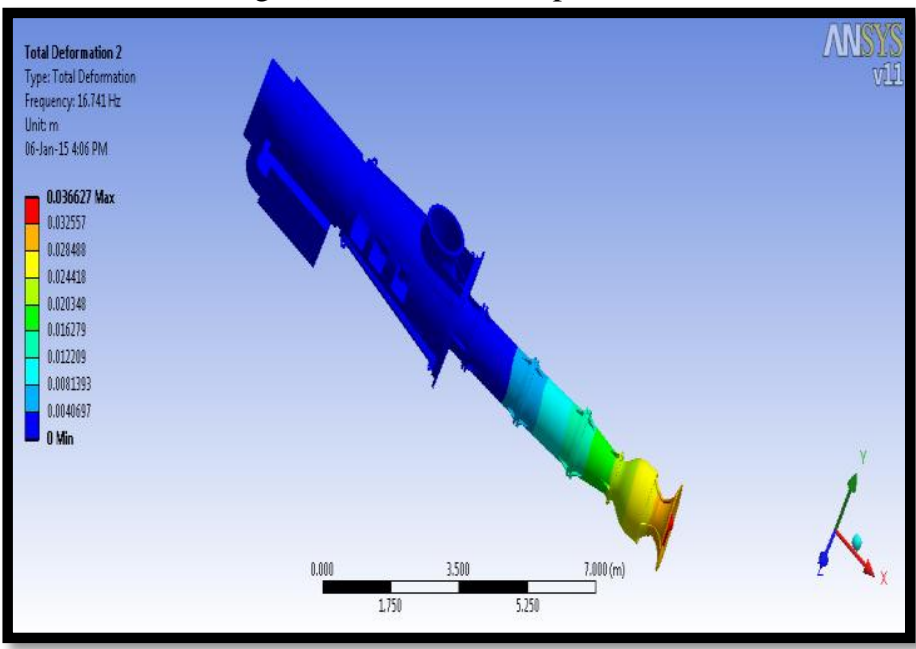

Fig 4(b) Second Mode shapes 
table 3. FEA results

\begin{tabular}{|c|c|}
\hline Mode Shape & Frequency \\
\hline 1 & 18 \\
\hline 2 & 25 \\
\hline 3 & 45 \\
\hline 4 & 59 \\
\hline 5 & 67 \\
\hline 6 & 85 \\
\hline
\end{tabular}

Minimum modal frequency found at mode first was approximately $2.34 \mathrm{~Hz}$. And maximum modal frequency was approximately $22.21 \mathrm{~Hz}$ at mode sixth.

III.

\section{EXPERIMENTAL MODAL ANALYSIS} (EMA)

Experimental Modal Analysis (EMA) is a type of impulseresponse test to determine the structural natural frequencies and the mode shapes of the pump system by using an instrumented hammer instead of natural excitation of the pump. It is a method of a meithod vibration testing in which a known force is put into a pump and pumps vibration response due exclusively to this force is observed and analyzed. EMA can determine the natural frequency of combined casing, piping, supporting structure. EMA can also determine the rotor natural frequency at the pump operating conditions.

\section{A. Impact Testing:}

Impact testing is conducted using two channel FFT spectrum analyzer, a micro computer with special software, a set of vibration response probes such as accelerometer, and an impact hammer designed to spread force over a frequency range that covers the test range.

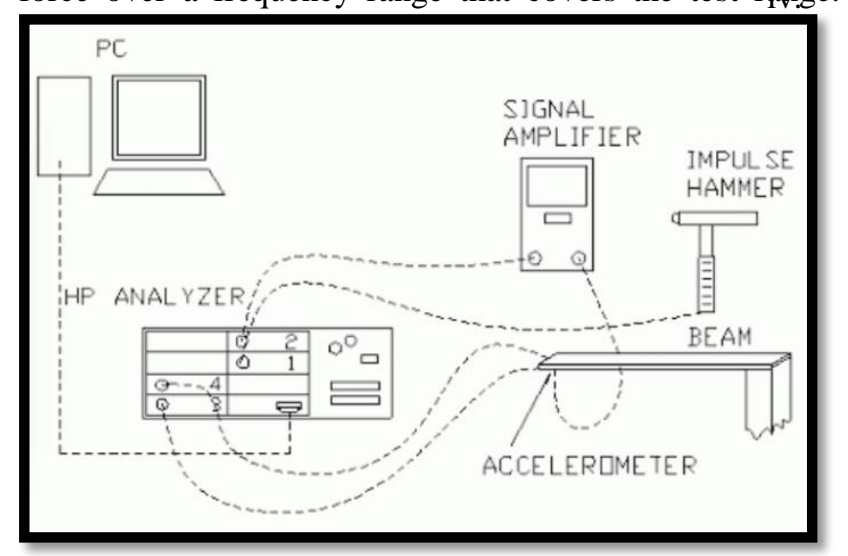

Fig. 5 Block diagram of basic Impact Testing

\section{Test Procedure:}

\section{B. Instruments used in Testing}

i. Accelerometer: An accelerometer is a device (transducer) which when attached to a vibrating structure gives out electric signal proportional to acceleration. This signal is given to the vibration analyzer which processes and analyzes the signal. In case of this experiment, the signal coming from accelerometer fitted to the structure is analyzed with respect to the one received from the impact hammer in order to find the natural frequency of the pump.

Impact Hammer: Impact hammer is used to excite the structure which is under vibration testing. This looks like an ordinary hammer but its head is fitted with a load cell and contains electronic circuitry and an output cable that can be connected to vibration analyzer, hitting the impact hammer on any structure an impulsive force is applied to the structure which is motor stool surface in our case. An equal and opposite force is sensed by the load cell fitted in the head of hammer, This generate an electric signal that is given to vibration analyzer which analyzes the signal, compares with the signals received from accelerometer fixed to the structure, and this information is used to develop FRF (frequency response function) and finally the natural frequencies of the structure found.

Vibration Analyzer: It is an electronic device that processes and analyzes the signals received from the transducer used in vibration measurement like impact hammer, accelerometer, digital tachometer, etc. It has numbers of channels, i.e. it can receive number of electric signals simultaneously. Four channel vibration analyzer is quite common. The vibration analyzer has very sophisticated electronic circuits and works together with a computer. Fast Fourier Transform (FFT) is an algorithm frequently used for analysis of the electric signals which provide frequency components and their corresponding amplitudes present in the signals.

A PC or a Laptop loaded with a software for modal analysis: In addition to the software used along with the vibration analyzer, software are available now a day devoted exclusively to the modal analysis. Such software facilitates inputting data to the computer related to geometry of the structure, location of fixing of the accelerometer on the structure as well as points of hitting of the impact hammer, their direction and so on. Also, there are facilities to select type of analysis required, ranges of various parameters of interest and so on. Such a software essentially process the signals received from the impact hammer and accelerometer, carrying out their FFT's, finding FRF, obtaining Mode Indicator Function, carrying out curve fitting over a selected range of frequencies, and finally providing the natural frequencies, mode shapes and modal damping factors.

In an impact test, an accelerator is fitted at some point on the structure and the structure is hit with an impact hammer giving an impulse to the structure. An engineered impact hammer is provided with different materials to be placed at its tip depending on the frequency response that is required (harder tips excite higher frequency). The tip 
should be selected such that the natural frequency of interest would fall within frequency span before first roleoff. Typically, is the machine is not operating; the impact number per test is about 10 to 15 . FFT analysis is done with the help of the signal received from the load cell fitted at the tip of the impact hammer. During an EMA test, the signal from hammer input force accelerometer is sent to one channel of spectrum analyzer, and the signal from the vibration response probes is sent to the second channel. Dividing, at each frequency, second channel by the first channel gives the frequency response function (FRF) of the pump and its attached system. The peaks of the FRF are the natural frequency, and width and heights of peaks indicate the damping of each natural frequency.

Ratio of signals received from accelerometer and that of impact hammer is taken in frequency domain which is called as FRF (Frequency Responce Function).

Corresponding FRF's are computed in the softwares of modal analysis. Frequency response function is obtained and which is shown below by graph amplitude vs frequency taken from display screen.

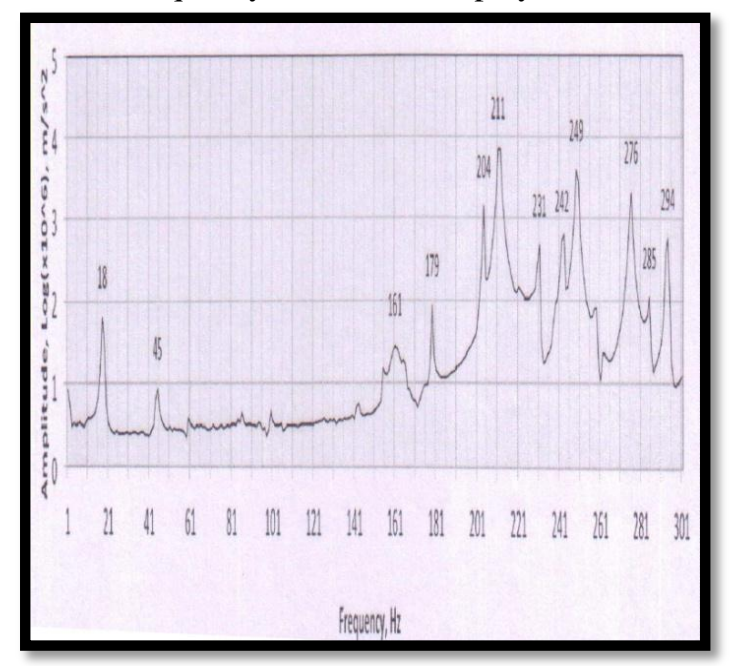

Fig. 7(a)

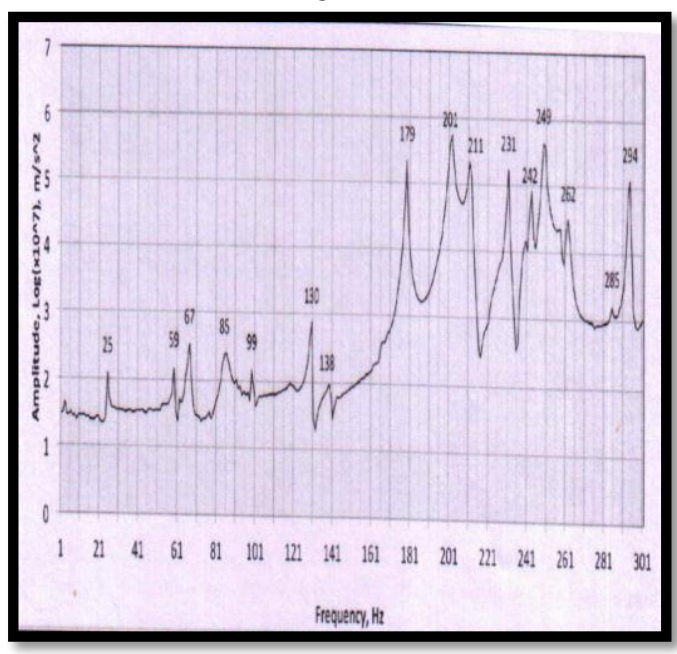

Fig. 7(b)
Modal identification is generated that shows peaks at the natural frequencies with the selected range of frequency.

By referring the above graph, we came to know that first natural frequency of VTP is $18 \mathrm{~Hz}$ which is greater than operating frequency, $12.3 \mathrm{~Hz}$. Hence, resonance is not occurring during the working of VTP. Mode shapes followed by their frequencies are tabulated below,

table 3. EMA results

\begin{tabular}{|c|c|}
\hline Mode Shape & Frequency \\
\hline 1 & 18 \\
\hline 2 & 25 \\
\hline 3 & 45 \\
\hline 4 & 59 \\
\hline 5 & 67 \\
\hline 6 & 85 \\
\hline
\end{tabular}

\section{CONCLUSION}

1) In this chapter main conclusion are drawn from the result obtained in this thesis.

The main objectives of this proposed work were:

i. To get natural frequency above $12.3 \mathrm{~Hz}$.

ii. To avoid resonance occur in VTP

2) To achieve this objective, in this thesis VTP was created by using Creo parametric 1.0 and FEA was done using ANSYS. The results obtained from ANSYS shows that the lowermost natural frequency is $16.667 \mathrm{~Hz}$.

3) This FEA results were compared with experimental results. The results obtained from EMA shows the lowermost frequency of $18 \mathrm{~Hz}$ which is quite similar to FEA results.

4) From the results and discussion it is concluded that the VTP is resonance free.

\section{REFERANCES}

[1] Paul Boyadjis and Chris Hurrel "Proactive planning with FEA" 2010

[2] Maki m. Onari,paul a. Boyadjis , "Solving structural and vibrational problem using FEA", 2009.

[3] J.E. Corley "Vibration problems of large vertical pumps" 1978.

[4] S.M. Abdel- Rahman, M.A.Hetal, M.A.Nasser "Vibrational probelems of axial flow pump".

[5] Vimalkumar A. Patel "Vertical turbine pump base stool modal analysis and model madification" 2013.

[6] Alejandro Pineda "Structural resonance problems on vertical pumps".

Fig. 7 Frequency Response Functions 